\title{
Retinal Image Enhancement Based on Contrast, Luminosity Adjustment and MSC
}

\author{
A.Anilet Bala, Pranav Pranshu Kanwar, Shipra Das, Debottama Das
}

\begin{abstract}
Retinal images have been widely used by ophthalmologists for detecting the retinal diseases before-hand and diagnosing them suitably. Old age macular degeneration, diabetic retinopathy and glaucoma are some examples of these diseases. However, poor quality of the image due to inadvertent circumstances limits the ability of the ophthalmologists to study the image. This paper hereby proposes an algorithm that is used to obtain clearer images by performing contrast and luminosity adjustment that enhances the basic quality of the clicked image. Following this, Multi-dictionary Sparse Coding (MSC) is carried out on the image to obtain the retinal vessel structures and miniscule details. Amount of Image enhancement is calculated by measuring the improvement after each stage of operation on the image. The image's quality is found to be much better compared to the other methods and thus can be suggested to the ophthalmologists for conducting the further medical studies conveniently.
\end{abstract}

\section{Keywords----RETINAL MULTI-DICTIONARY \\ IMAGE \\ SPARSE \\ CONTRAST-LUMINOSITY ADJUSTMENT, \\ LEARNING, SPARSE CODING}

\section{INTRODUCTION}

The quality of a retinal image is clinically unsatisfactory due to inevitable causes such as eye lesions and patient's eye movement. Because of inability of attaining cent percent focus, the resulting image is blur, non-uniformly illuminated with low contrast hence being an unreliable image for diagno-stic purposes. These images are of excessively low quality, have a low contrast and suffer deteriorated luminosity due to the imaging conditions and undergo color distortion. High quality retinal images are desirable for a myriad of applications. Some important ones being the study of retina, early detection of diseases, biological research.

Considering the importance and the need for high quality retinal images, a number of image processing techniques have been proposed. The goal is to obtain a better, detailed image of the retina that gives sufficient knowledge of the vessel structures. The various methods incorporate different

Revised Version Manuscript Received on 10 September, 2019.

A.Anilet Bala, Assistant Professor, Department of Electronics and Communication, SRM Institute of Science and Technology, Chennai, Tamil Nadu, India.(Email: shipratut96@gmail.com)

Pranav Pranshu Kanwar, UG Scholar, Assistant Professor, Department of Electronics and Communication, SRM Institute of Science and Technology, Chennai, Tamil Nadu, India.(Email: prn.007gt40@gmail.com)

Shipra Das, UG Scholar, Assistant Professor, Department of Electronics and Communication, SRM Institute of Science and Technology, Chennai, Tamil Nadu, India.(Email: anizimbra@ gmail.com)

Debottama Das, UG Scholar, Assistant Professor, Department of Electronics and Communication, SRM Institute of Science and Technology, Chennai, Tamil Nadu, India. set of advantages according to the need of the situation. However, each method has its own disadvantages too.

In one of the methods, histogram equalisation is used. This method is based on histogram fitting and stretching. T though the method produces a clearer and brighter image, the retinal vascular details are lost.[1] Another method which is based on contourlet transform results in the changing of some vascular parameters while undergoing transformation. This results in the loss of naturalness of the image. [2] The filter based method that usually uses a Gabor filter results in obtaining the vascular details but with distorted vessel structures.[3] To avoid the problem of uneven illumination in the retinal images, a method that comprises of contrast and luminosity normalization is used. This

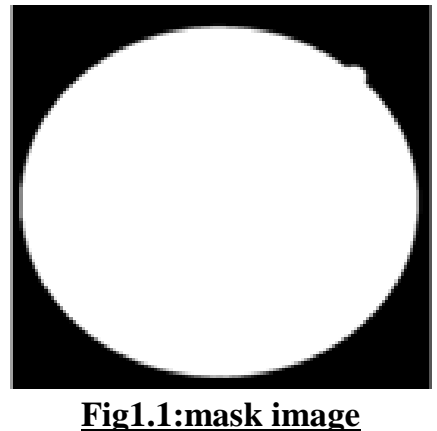

method though, hardly accounts for the loss of vascular details.[4]

Some of the other work related to retinal image processing includes the bowler hat transform a new multiscale vessel enhancement approach which is a mathematical morphology has been proposed.[10] The method combines different structural element to detect the vessels.Another method proposed is Contrast Limited Adaptive Histogram Equalization (CLAHE) for preprocessing and Tandem Pulse Coupled Neural Network (TPCNN) model for automatic feature vectors generation then classification and extraction of the retinal blood vessels based on Deep Learning Based Support Vector Machine (DLBSVM).[11]

In this paper, we propose an algorithm which is a combination of contrast, luminosity adjustment and multi-dictionary sparse coding(MSC).[5] This is a stepwise 


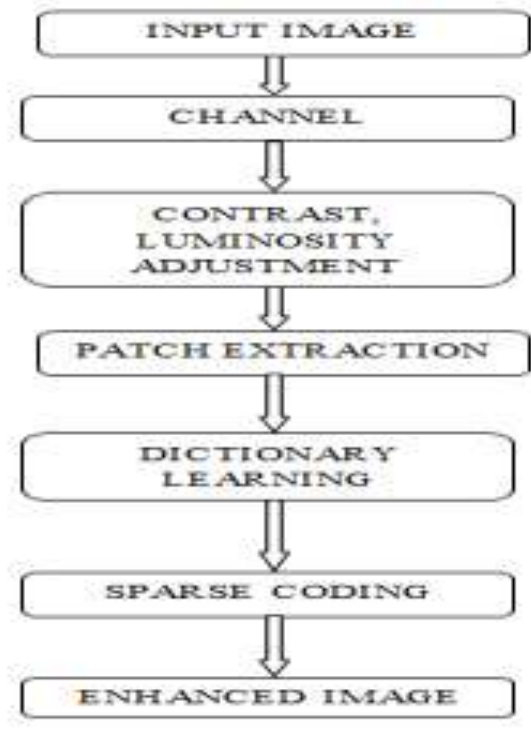

Fig2.1:Block Diagram

algorithm in which firstly, the illumination is taken care of by contrast adjustment and luminosity normalisation by CLAHE(Contrast Limited Adaptive Histogram Equalisation) respectively and secondly, the vascular details and structures [6]

In MSC, Representation Dictionary (RD) and Enhancement Dictionary(ED) are put into use for obtaining the detailed structure of the vessel. RD is generated from the original vessel images and ED from the corresponding label Sparse coding technology. This enables the computation of the sparse coefficients. Post that, sparse coefficients along with ED reconstruct the enhanced target image. At last, we rescale the enhanced image to get the final result. For comparing the performance of the proposed method with the various approaches hitherto, we collected the results published in the past years in reputable journals and conferences.

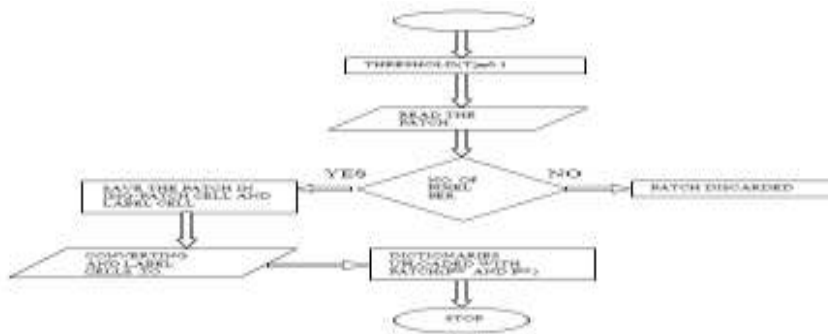

Fig2.2: Patch selection

In this paper there are three sections.The next section consists of the system methodology followed by result and conclusion.

\section{SYSTEM METHODOLOGY}

The original retinal image is a three dimensional image with RGB components. To obtain the retinal part in isolation from the background for closely studying the retina, masking is done. For this, masks are obtained from the research centre and gamma correction (in the luminance gain matrix) are obtained through dictionary learning and sparse coding. images. Vessel image that is targeted is then depicted by

\section{A. Masking Process}

too, as shown in fig1. As evident in the figure, masks are two-dimensional images that are represented only by binary values.

The redundant background of the image is separated out by taking the product of the input image and its corresponding mask image. The redundancy is removed by approximately ninety percentages at this step.

The required part is obtained by matrix multiplication as follows:

Required Image $=$ Original Image $\mathrm{x}$ Mask Image

\section{B. Contrast Adjustment}

For enhancing the contrast of retinal images, the CLAHE (Contrast Limited Adaptive Histogram Equalisation) method is applied. CLAHE divides the image into small regions called tiles: local contrast is enhanced by equalising the corresponding histogram for each tile. . For noise superseding in the local region,clip limitation strategy is used . It is preferable to execute CLAHE on the luminosity channel to avoid distortion of colour. This technique for contrast improviasation works only on the luminosity channel. Henceforth, the retinal image in RGB color space by is converted into $\mathrm{L}^{*} \mathrm{a} \mathrm{b}^{*}$ color space. The CLAHE then enhances the L channel. Finally, the image, after being processed, is transformed back into the RGB color space. from the $\mathrm{L} * \mathrm{a} * \mathrm{~b} *$ color space.

\section{Luminosity Enhancement}

For enhancing the luminosity with preservation of color and naturalness, the R, G and B channels should be modified by the same proportion. Luminance gain matrix $\mathrm{G}(\mathrm{x}, \mathrm{y})$ is defined as follows:

$$
\frac{r^{\prime}(x, y)}{r(x, y)}=\frac{g^{\prime}(x, y)}{g(x, y)}=\frac{b^{\prime}(x, y)}{b(x, y)}=G(x, y)
$$

To find the color-independent luminance gain matrix, the colour image is transformed into the HSV colour space where the luminosity channel $(\mathrm{V})$ is decoupled from the other two color components: hue $(\mathrm{H})$ and saturation $(\mathrm{S})$. Maximum ( $\max$ ) of the R, G, and B values gives the luminance intensity of a pixel at the $(\mathrm{x}, \mathrm{y})$ position:

$$
G(x, y)=\frac{V^{\prime}(x, y)}{V(x, y)}=\frac{V^{\prime}(x, y)}{\max (r(x, y), g(x, y), b(x, y))}
$$

\section{Denoising by energy minimisation}

The image post doing contrast, luminosity adjustment is sent for dictionary learning after patch segregation and selection. Sparse coding is done along with dictionary learning. The basic denoising function is given as follows:

$$
\begin{array}{r}
\frac{1}{2}\|X-Y\|_{2}^{2}+G(X), \\
\text { where y: noisy image } \\
\mathrm{x}: \text { clean image } \\
\mathrm{G}(\mathrm{x}): \text { prior function }
\end{array}
$$

The prior function, $\mathrm{G}(\mathrm{X})$ gives the constraints to be followed during denoising the image. The sparse prior is given as: $\quad \mathrm{G}(\mathrm{X})=\lambda\|\alpha\|$, for $\mathrm{X}=\mathrm{D} \alpha$

where $\mathrm{D}$ is the dictionary and $\alpha$ is the sparse coefficient.

\section{Published By:}

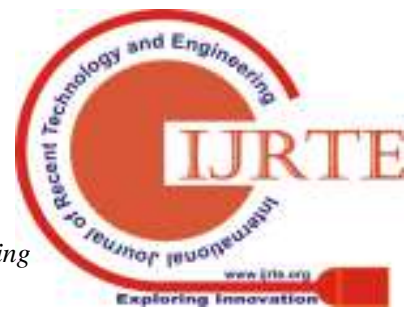




\section{E.Patch Selection}

The image is first divided into patches. The patches that belong to background now have to separate out by setting a threshold ( 0.1 here) for the number of pixels per patch.Refer fig 2.2.

\section{F. Sparse Dictionary Learning}

A patch, $\mathrm{X}$ is said to admit to sparse approximation on a dictionary, $\mathrm{D}$ in where $\mathrm{k}$ (atoms) is the no. of columns, when a linear combination of a few atoms(from $\mathrm{D}$ ) that are close to $\mathrm{X}$ can be found. If $\mathrm{D}$ is excessively large,it results in arbitrarily low values of $\alpha$. To avoid this, it columns are limited to have an $\ell 2$ norm less than or equal to one,i.e. Euclid distance of each patch is set to be 1 or less than one by normalisation.

\section{G. Proposed Algorithm}

The algorithm is used to implement the sparse dictio learning on the test images (contrast, luminosity enhanced) to get enhanced images. The steps are as follows: The single dictionary is separated into representation and enhancement dictionary by separating the first half from the second half. Since we have selected the patch size to be $5 \times 5$, the image size has to be a multiple of 5 . If not, the image is resized by calculating the remainder. Overlapping pixels in two patches are found. For each patch, no. Of pixels sharing the patches are found. Mean of these pixels are subtracted from the patch, since mean is the DC component. Sparse coefficients are generated.

Sparse coefficients x Dictionary = Enhanced Dictionary,

By Matrix Multiplication Convert to 2D. Add the mean back to the output.

The Results section shows the outputs for the same.

\section{RESULTS AND DISCUSSIONS}

The following outputs are obtained on applying the above algorithm. In table 1 the PSNR value of enhanced image using proposed method is 64.62.

It shows the quality of the fundus image is good for further medical analysis.

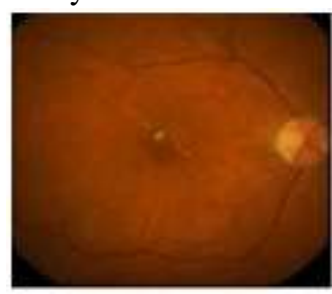

(a)

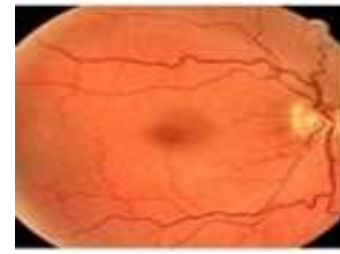

(c)

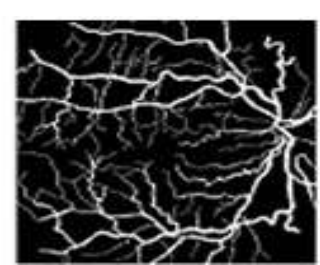

(b)

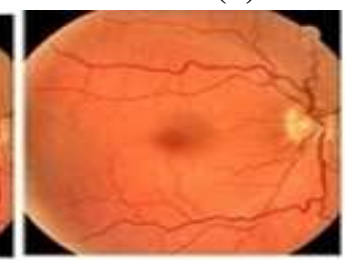

(d) a) Original image b) label image c) enhanced image using contrast, luminosity adjustment d) enhanced images proposed method

\begin{tabular}{|l|l|l|}
\hline MSE & PSNR & NCC \\
\hline
\end{tabular}

\begin{tabular}{|l|l|l|}
\hline 0.048 & 64.62 & 0.891 \\
\hline \multicolumn{3}{|c|}{ Table 1: Performance Analysis for sample } \\
\hline
\end{tabular}

\section{CONCLUSION}

Here an effective method for color retinal image enhancement based on a combinative algorithm of contrast, luminosity adjustment and multi-dictionary sparse coding has been proposed. Experimental results show that the proposed method not only improves the poor illumination (contrast, luminosity) but also gives information of the miniscule vascular details. These vessel structures can, in future, be compared to that of basic diseased retinas to warn the patient immediately. The superiority of this method is mainly contributed to the undertaking of dictionary learning and sparse coding post contrast adjustments. By using the dictionary learning technology, we can not only achieved better results but also save much more time for the patients as sparse coding lessens the complexity hence reducing the time of computations

\section{REFERENCES}

1. M. D. Abramoff et al., "Retinal imaging and image analysis," IEEE Rev. Biomed. Eng., vol. 3, pp. 169-208, Dec. 2010.

2. A. F. M. Hani and H. A. Nugroho, "Retinal vasculature enhancement using independent component analysis," J. Biomed. Sci. Eng., vol. 2, no. 7, pp. 543-549, Nov. 2009.

3. J. Paulus et al., "Automated quality assessment of retinal fundus photos,” Int. J. Comput. Assist. Radiol. Surg., vol. 5, no. 6, pp. 557-564, Nov. 2010.

4. U. Sevik et al., "Identification of suitable fundus images using automated quality assessment methods," J. Biomed. Opt., vol. 19, no. 4, p. 046006, Apr. 2014.

5. M. R. K. Mookiah et al., "Computer-aided diagnosis of diabetic retinopathy: a review," Comput. Biol. Med., vol. 43, no. 12, pp. 2136-2155, Dec. 2013.

6. M. R. K. Mookiah et al., "Computer-aided diagnosis of diabetic retinopathy: a review," Comput. Biol. Med., vol. 43, no. 12, pp. 2136-2155, Dec. 2013.

7. E. Daniel and J. Anitha, "Optimum green plane masking for the contrast enhancement of retinal images using enhanced genetic algorithm," Optik, vol. 126, no. 18, pp. 1726-1730, Sep. 2015.

8. M. Foracchia et al., "Luminosity and contrast normalization in retinal images," Med. Image Anal., vol. 9, no. 3, pp. 179-190, Jun. 2005.

9. P. Feng et al., "Enhancing retinal image by the Contourlet transform," Pattern Recogn. Lett., vol. 28, no. 4, pp. 516-522, Mar. 2007.

10. Sazak, Çiğdem, Carl J. Nelson, and Boguslaw Obara. "The multiscale bowler-hat transform for blood vessel enhancement in retinal images." Pattern Recognition 88 (2019): 739-750. 